\title{
ANÁLISE DA PERCEPÇÃO DOS IDOSOS INTEGRANTES DE GRUPO DE PRÁTICAS CORPORAIS NA ATENÇÃO PRIMÁRIA: aspectos motivacionais e o fazer multiprofissional
}

\author{
Braulio Nogueira de Oliveira' \\ Wellington Gomes Feitosa ${ }^{2}$ \\ Heraldo Simões Ferreira ${ }^{3}$
}

\begin{abstract}
RESUMO
O objetivo deste artigo foi analisar as percepções dos idosos, acerca dos aspectos motivacionais e nível de significância/importância das práticas corporais, bem como do trabalho multiprofissional desenvolvido pela equipe de saúde. O estudo caracterizase como descritivo e exploratório, com abordagem qualitativa, em que se utilizou o método da análise de conteúdo. Foram entrevistados 14 idosos. As medidas terapêuticas, atreladas a alguns resultados positivos e a promoção da saúde de maneira geral foram os principais motivos elencados. Quanto ao fazer multiprofissional notou-se atuação interdisciplinar pouco efetiva. Diante disso, uma ressignificação desses espaços se faz necessária.
\end{abstract}

Palavras-chave: Exercício; Promoção da Saúde; Saúde do Idoso.

1 Graduado em Educação Física (Universidade Estadual do Ceará), Especialização em andamento em Saúde do Idoso (Universidade Estadual do Ceará), Especialização em andamento em caráter de Residência Multiprofissional em Saúde da Família (Universidade Estadual Vale do Acaraú) Contato: brauliono08@homail.com.

2 Graduado em Educação Física (Universidade Federal do Ceará). Especialização em Fisiologia e biomecânica dos movimentos, Especialização no ensino de educação física e Especialização em Personal Training, Mestrado em andamento em Ciências do Desporto com especialização em Avaliação e Prescrição na Atividade Física (Universidade de Trás-os-Montes e Alto Douro). Contato: wellfitness@hotmail.com.

3 Graduado em Educação Física (Universidade de Fortaleza), Especialista em Psicomotricidade (Universidade Estadual do Ceará), Mestre em Educação em Saúde (Universidade de Fortaleza), Doutor em Saúde Coletiva (Universidade Estadual do Ceará).Contato: heraldosimoes@bol.com.br. 


\section{INTRODUÇÃO}

O Brasil passa por uma transição demográfica e epidemiológica acentuada. Esse processo se caracteriza por uma redução da mortalidade infantil e da natalidade com consequente aumento percentual da população idosa, além de uma redução da prevalência das doenças infecciosas e um concomitante aumento das doenças e agravos não transmissíveis.

Nesse contexto, destacamos a Política Nacional de Promoção da Saúde, que emerge com o objetivo de promover a qualidade de vida e reduzir vulnerabilidade e riscos à saúde relacionados aos seus determinantes e condicionantes (MALTA et al., 2009). Essa política institucionaliza a promoção da saúde no Sistema Único de Saúde (SUS) e elenca a atividade física/ práticas corporais enquanto uma de suas prioridades (MALTA et al., 2009).

A partir do entendimento de promoção da saúde pautado na integralidade da atenção, outras categorias profissionais foram inseridas no âmbito da Atenção Primária à Saúde, no sentido de contemplar essas demandas. Nessa feita, os Núcleos de Apoio à Saúde da Família (NASF) surgiram com uma proposta de ampliar a abrangência e o escopo das ações e devem atuar integrada a rede de serviços em saúde com acompanhamentos longitudinais, com intuito de contribuir no apoio as Equipes de Saúde da Família em territórios definidos (BRASIL, 2008).

Assim como a Política Nacional de Promoção da Saúde, uma das ações prioritárias do NASF é justamente as atividades físicas/ práticas corporais, devendo desenvolver essas atividades em beneficio da comunidade. Um diferencial do fazer desses profissionais, deve ser a atuação interdisciplinar, atrelado a ações educativas e coletivas, superando as práticas puramente clínicas. Sabe-se que grande parte dessa atuação se dá por meio de atividades sistemáticas grupais e em geral contempla muitos idosos.

Segundo Luz (2007), há uma tendência no cenário brasileiro de conscientização em relação à relevância social e dos próprios benefícios físicos do exercício físico. Essas práticas devem superar o paradigma cartesiano, passando a ter uma maior significação social, visto que trata-se de espaços de convivência e compartilhamento de situações eminentemente passíveis de ações transdisciplinares.

Muitos estudos remetem à necessidade de uma formação diferenciada em se tratando da Saúde do Idoso (DIOGO, 2004; MOTTA; CALDAS; ASSIS, 2008; GUIMARÃES, 1987). De fato, há muito tempo existe uma preocupação em relação às políticas para proteção e saúde do idoso, incluindo medidas gerais de promoção da saúde, prevenção de agravos à Saúde, assistência à saúde diferenciada para esse público (GUIMARÃES, 1987).

Diante da complexidade da atuação no NASF e de toda a conjuntura de assistência ao geronte, o presente estudo pretende analisar as percepções dos próprios idosos, acerca dos aspectos motivacionais e nível de significância/ importância desses espaços para a promoção da sua saúde, além do trabalho multiprofissional desenvolvido pela equipe de saúde.

\section{METODOLOGIA}

O presente estudo caracteriza-se como descritivo e exploratório, com 
abordagem qualitativa que pressupõe contato mais aprofundado com os diversos saberes e percepções dos sujeitos envolvidos, além de possibilitar maior aproximação com o cotidiano e as experiências vividas pelos próprios (MINAYO, 1993). Segundo Gil (1994), o modelo descritivo tem por objetivo principal a descrição das características de determinada população ou fenômeno, ou estabelecimento entre determinadas variáveis.

Foram realizadas entrevistas com 14 idosos, pertencentes a dois grupos em que os profissionais do NASF são cuidadores em Fortaleza/CE, 12 idosos eram do gênero feminino e apenas dois do gênero masculino. A média de idade da amostra foi de 71 anos. Todos deveriam ter idade superior a 60 anos e participação nas atividades do NASF há, no mínimo, três meses.

A coleta de dados ocorreu entre os meses de outubro de 2011 a janeiro de 2012. A entrevista foi gravada com o auxílio de um aparelho digital (Mp4 Foston - Fs 66) e posteriormente transcrita para a realização da análise.

Para atingir os resultados almejados, foi utilizado o método denominado "Análise de Conteúdo". Esse método propõe encontrar respostas para os questionamentos formulados, além disso, diz respeito à descoberta dos elementos inerentes ao conteúdo manifesto (BARDIN, 1977).

Segundo Bardin (1977) a análise de conteúdos do tipo análise temática desdobra-se em três fases: a primeira corresponde à fase de pré-análise que se consiste na escolha e organização do material a ser analisado, constituindo-se pelas tarefas: leitura flutuante, constituição do Corpus e formulação de Hipóteses e objetivos; a segunda é a fase da exploração do material que se resume em realizar a codificação, ou seja, aqui é o momento de se aplicar o que ficou definido na fase anteriormente mencionada, podendo ser necessário realizar inúmeras leituras de um mesmo material. $\mathrm{Na}$ terceira fase efetuamos o tratamento dos dados obtidos e sua interpretação, visando desvendar o conteúdo que está nas entrelinhas do que está sendo manifestado, sem excluir as informações estatísticas, sendo importante se voltar para ideologias e tendências que descrevem bem os fenômenos analisados.

Foi sucintamente explicado aos participantes os objetivos e garantia dos princípios éticos por parte do entrevistador que são vigorados pela Resolução 196/96 do Conselho Nacional de Saúde que norteia as pesquisas que envolvem seres humanos. Nesse sentido, O projeto foi analisado e aprovado pelo Sistema Municipal de Saúde Escola de Fortaleza/CE, bem como pelo Comitê de Ética em Pesquisa da Universidade Estadual do Ceará (processo n. 11222606-0).

\section{ASPECTOS MOTIVACIONAIS E A CONS- TRUÇÃO DE VÍNCULOS COM O GRUPO}

Os idosos foram questionados a respeito do principal motivo da sua participação nas atividades do grupo, tendo sido relatados pelos mesmos, principalmente, por medidas profiláticas específicas, atreladas a alguns resultados positivos e pela promoção da saúde de maneira geral, que inclui a criação de vínculos e a participação social. Sabemos que, seja qual for(em) o fator(es) motivacional(ais) para esses idosos, a simples convivência social propiciada pelo grupo, torna-se relevante para que se obtenha bons níveis de saúde, pautada no 
princípio da integralidade relacionada às práticas de saúde. Como pode ser notado nas falas a seguir, há idosos que participam principalmente por motivos terapêuticos:

Por que eu tenho dor nas pernas, pressão alta. Faço caminhada. (Entrevistado 12) Por causa do meu problema da coluna que eu não tô podendo andar. Começa a doer daqui do cóccix. (...). (Entrevistado 9)

Vale ressaltar que a maior parte dos idosos participa por motivos voltados para a prevenção e tratamento de determinadas patologias e agravos, embora, o papel da Estratégia Saúde da Família, contemple vários outros aspectos e a própria patologia possui determinantes sociais pertinentes. Nesse sentido, alguns idosos se avaliam em significante melhora após a participação no grupo, muito embora não abordássemos essa questão:

É muito importante. Eu era uma pessoa inutilizada. Eu não me acocava pra pegar nem uma colher que caísse no chão. Hoje eu me deito no chão, me sento no chão, me levanto sem ajuda de ninguém. (Entrevistado 10)

Ahh, por causa dos meus ossos que estavam tudo assim né, com dores nas juntas, aí eu vim fazer. Melhorei muito depois que eu comecei. E eu gosto! Eu gosto de fazer atividade, alongamento. (Entrevistado 13)

Segundo Czeresnia (1999), a prevenção trata de ações voltadas para evitar o surgimento de doenças específicas, com diminuição de sua incidência e prevalência nos usuários. A autora conceitua a promoção da saúde como sendo mais amplo, tendo em vista que esta se refere a mudanças na própria condição de vida das pessoas, relacionando-se a melhoria de uma maneira geral e bem-estar em suas diversas dimensões. Desse modo, a prevenção é uma ferramenta da promoção da saúde, assim como a própria Estratégia Saúde da Família. A partir das falas dos idosos, percebemos também motivos voltados para a promoção da saúde em seu sentido mais abrangente, ou seja, não voltadas apenas para práticas curativas ou preventivas:

Assim, eu acho que agente se envolver, se engajar, estar junto. É importante agente saber que tem alguma coisa a ver com o outro. compartilhar, saber o que estar acontecendo com o outro, o outro saber o que está acontecendo com a gente, assim, as coisas do dia-a-dia a rotina do dia-a-dia, vamos dizer assim. Que eles saibam o que eu sou e o que eu sinto e que eu saiba, né!? coisas desse tipo. Os exercícios são muito importante. Essas coisas que eles nos ensina, que é muito bom até pra o físico da gente, os exercícios, assim, desenvolver alguma coisa que agente não tem tempo de caminhar né? Fazer caminhada, e daí isso é muito bom pra gente. (Entrevistado 2)

Em estudo realizado em Natal-RN, foi analisada a percepção dos idosos acerca das representações sociais da atividade física nesse caso, foram relatadas a dimensão psicológica, social e biofísica. Além disso, notou-se grande ênfase no termo felicidade (SANTANA; MAIA, 2009). Desse modo, percebe-se uma abordagem biopsicossocial no que se refere à saúde do idoso. Fato como esse evidencia a atividade física como um instrumento diferencial para promoção da saúde, e não somente para a prevenção e/ou tratamento de doenças e agravos.

A ideia de promoção da saúde, no sentido ressaltado pela Czeresnia (1999), 
relaciona-se diretamente com um dos sentidos da integralidade proposto por Mattos (2004) e Machado, et. al. (2007). Segundo esses autores, é preciso que o cuidado com o usuário seja pautado em práticas intersubjetivas, tendo como ferramenta projetos terapêuticos singulares. Para isso, é preciso que se tenha uma postura, por parte dos profissionais do NASF e da equipe de Saúde da Família que permeiem a transdisciplinaridade, não restringindo sua atuação ao caráter multidisciplinar, sendo essa prática em demasia considerada falha por alguns autores (DIOGO, 2004; MOTTA; CALDAS; ASSIS, 2008; CAMPOS; BELISÁRIO, 2001; SILVA; ROS, 2007; ANJOS; DUARTE, 2009). Desse modo, é preciso "desconstruir" uma maneira de atuação pautada na formação acadêmica tradicional e individual para "construir" novas formas de intervir em equipe.

Os resultados foram unânimes no que se refere à importância, atribuída pelos idosos, aos grupos de práticas corporais. Talvez, e meramente supondo, tal atribuição seja justificada pelos benefícios propiciados pelas vivências no grupo. Segue os relatos decorrentes:

É muito importante! Não é importante não, é muito importante viu! Isso aí não tá fazendo mal não, tá é ajudando!" (Idoso 8)

Acho! Muito importante! Olha, esses profissionais da saúde estão de parabéns por que estão se preocupando mais com a terceira idade tá entendendo? Pela saúde do povo sabia? Isso é muito importante. Isso deveria ser um dia e outro não né? E não só uma vez por semana. (Idoso 1)

A falta de espaços de interação e convívio entre as pessoas idosas e as demais faixas etárias é preocupante. Percebe-se que os idosos consideram esses espaços importante pelo simples convívio e relações sociais promovidas de maneira direta ou indireta pelo grupo, suprindo uma carência intrínseca, além de desvincular, as práticas corporais ao exercício físico puramente utilitarista da redução de fatores de risco por exemplo. Ramos (2002) mostra haver um maior bem-estar nos idosos ao conviverem com conjugue ou terceiros, em relação aos que convivem com filhos ou parentes. Não obstante, ainda ao questionarmos a importância dos espaços destinados as práticas corporais, nota-se também no relato dos idosos a questão da espiritualidade muito forte:

Importante demais! Demais! Ave Maria, a Educadora Física é muito boa, o que ela faz com agente é muito bom. Quinta-feira eu fiz tanto exercício que de noite eu estava tão aliviada. (Entrevistado 2)

É ótimo! É ótimo! Ave Maria, eu conheço a Educadora Física faz mais de um ano que eu a encontro lá no (...) e ela já ia pra lá fazer essas atividades educativas, mas lá só é uma vez por mês, e aqui é mais, graças a Deus. (Entrevistado 3)

Segundo Negreiros (2003), nada pode ser afirmado a respeito do fato de os idosos estarem com a espiritualidade mais presente que em outras fases da vida, no entanto, a autora acredita ser decorrente de um desejo maior por uma vida eterna, ou até mesmo por atingirem um nível de maturidade que permite delimitar melhor o sentido da própria vida, e desse modo, aceitar a morte. Supõe ainda, que um postulado possa estar atrelado ao outro, engendrando uma motivação bem maior que caso fossem isolados. 
Diante do exposto evidencia-se uma realidade multifacetada das percepções dos idosos acerca dos grupos de práticas corporais, visto que trata-se de um espaço sucumbido de relações intersubjetivas e construção de vínculos, superando o paradigma cartesiano do exercício físico.

\section{O FAZER MULTIPROFISSIONAL NOS GRUPOS DE PRÁTICAS CORPORAIS}

No sentido de refletir as práticas do NASF nos grupos de idosos, foram analisadas as percepções acerca do trato multiprofissional nas intervenções do grupo. Desse modo, ao questionar acerca da inserção de outras categorias profissionais além do profissional de Educação Física nesses espaços, encontraram-se os seguintes relatos:

Não ela trabalha com outra, mas eu não estou lembrada o nome da outra, uma baixinha. Ei, como é o nome daquela outra Doutora? Não estou lembrada. (Entrevistado 3)

Ela faz os exercícios sozinha, mas tem essas outras que ajudam né? (Entrevistado 10)

Não, só ela mesmo. Vem duas mocinhas com a gente mais só quem ensina a gente é ela. Eu acho que são amigas dela. (Entrevistado 5)

Não obstante, note-se uma participação pouco efetiva por parte dos demais profissionais do NASF nos grupos de práticas corporais. Possivelmente também não ocorra de maneira significativa a atuação do profissional de Educação Física nos grupos não voltados para Atividade Física/ Práticas Corporais. Desse modo, tornam-se necessárias ações de educação permanente voltadas para a capacitação dos trabalhadores em saúde, acerca da promoção de intervenções de cunho interdisciplinar, consolidando, de fato, a atuação multiprofissional da equipe. Silvestre e Costa Neto (2003) propõem atribuições comuns para os integrantes da equipe de Saúde da Família em relação à saúde do idoso, que embora não sejam específicas da equipe multiprofissional do NASF, podem de certa forma, nortear sua atuação. Entre elas podemos destacar o conhecimento da realidade das famílias, a identificação dos problemas de saúde e situações de risco mais comuns, valorização das relações com a pessoa idosa e sua família, a realização de visitas domiciliares de acordo com o planejado, prestação de assistência integral, garantia do acesso ao tratamento dentro de um sistema de referência e contra-referência, coordenação e participação e/ou organização de grupos de educação para a saúde além de promoção de ações intersetoriais.

Para além das ações postuladas por Silvestre e Costa Neto (2003), novas formas de intervenção estão surgindo, no sentido de possibilitar e viabilizar maios resolutividade no serviço, bem como propiciar novas perspectiva consolidar a promoção da saúde. Miranda e Farias (2009), por exemplo, relatam o uso da internet como um meio de contribuir positivamente para o bem-estar do idoso, tanto no que se refere ao seu caráter informativo, quanto em seu caráter lúdico, relacionando-se diretamente com a prevenção da depressão, manutenção dos níveis cognitivos, e redução do isolamento social, principalmente para os limitados fisicamente.

Todavia, como podemos perceber nas falas abaixo, o apoio prestado pelos Agentes Comunitários de Saúde (ACS) ocorre de maneira substancial. Sabemos que um papel importante no processo de 
consolidação da Estratégia Saúde da Família vem sendo de responsabilidade desses profissionais. Estes são os principais responsáveis pelo elo existente entre a comunidade e as unidades de saúde, além de serem os representantes da equipe mínima de Saúde da Família. Desse modo, o contato direto com a comunidade é papel principalmente desses profissionais, sendo em potencial, um diferencial em se tratando de propiciar subsídios para consolidação do trabalho em saúde.

Ela atua junto com os agentes de saúde, pra qualquer coisa que precisar. (Entrevistado 1)

Ela trabalha junto com as outras moças aqui do posto, com as agentes de saúde né? (Entrevistado 6)

Em estudo acerca da saúde do idoso percebida pelos ACS, grande parte deles, atribuíram ao conceito de saúde do idoso uma visão de integralidade da atenção, todavia, percebeu-se, por parte de alguns desses profissionais, uma visão negativa a respeito da representação social do idoso, direcionados à presença de enfermidades, dependência física e emocional (BEZERRA; ESPIRITO SANTO; BATISTA FILHO, 2005). Nesse sentido, ao serem consideradas as opiniões dos idosos nas visitas domiciliares, os problemas de doenças são considerados prioritários, sendo relatados ainda a falta de atenção, solidão e isolamento do contexto familiar.

Faz-se sempre presente nas percepções dos ACS a respeito da saúde do idoso, fatores relacionados com a saúde mental. Rodrigues e Araujo (2010), por exemplo, identificaram em Vale do Paraíba-SP, relatos dos ACS em que todos se referem a queixas dos idosos permeiam a solidão, falta de atenção dos familiares; além de: problemas financeiros e espera por consultas e exames.

Os agentes são acompanhados e orientados por um enfermeiro, o qual atua como instrutor-supervisor. No entanto, é possível, com a inserção de novos profissionais de saúde enquanto apoiadores matriciais, promovendo um trato interdisciplinar de saberes, criando novas perspectivas ao empoderamento das ações em saúde pautada na educação permanente que viabiliza uma troca de saberes a partir dos processos de trabalho.

\section{CONSIDERAÇÕES FINAIS}

A inserção de outras categorias profissionais no apoio à Saúde da Família, a inserção das atividades físicas/ práticas corporais enquanto uma das prioridades além da institucionalização da promoção da saúde no SUS são medidas estratégicas relevantes e devem evoluir pari passu a transição demográfica e epidemiológica se consolida no cenário nacional.

Decerto, essas práticas não contemplam apenas aspectos inerentes a melhorias na aptidão físico/funcional dos idosos, mas influenciam diretamente na promoção da saúde dessas pessoas. Portanto, podemos considerar que influenciam na adesão e manutenção dessas atividades os aspectos socioculturais, políticos e educativos inerentes à própria cultura corporal desenvolvida com o passar dos anos no geronte.

Os motivos terapêuticos foram prevalentes no presente estudo, e geralmente estavam atrelados a resultados positivos, como a redução de dores e a própria recuperação de algumas capacidades funcionais relacionadas às atividades de vida diária. Além disso, encontraram-se resultados direcionados a promoção da saúde em seu sentido 
ampliado, compreendendo a importância de um espaço destinado ao compartilhamento de sentimentos e consequente construção de vínculos. A conscientização da importância dos exercícios físicos foi ressaltada, possivelmente fruto do positivo trabalho multiprofissional desenvolvido. Embora não tenha sido foco do estudo, a espiritualidade se mostrou muito presente nos idosos.

Entende-se que o trabalho multiprofissional pode contribuir imensuravelmente nas práticas grupais, todavia, a categoria Educação Física, além dos ACS foram praticamente às únicas notadas nos relatos dos idosos, seja pelas intervenções não ocorrerem de maneira multidisciplinar; por ocorrerem com pouca frequência ou significância ou por inexistirem. Nesse sentido, é preciso que se tenha a clareza de identificar as demandas pertinentes a cada núcleo de saber do NASF para que seja direcionada a intervenção específica interdisciplinar, além de estreitar laços com a equipe mínima de Saúde da Família (além dos ACS).

Diante do exposto entendemos que as atividades físicas/ práticas corporais devem ser resignificadas, no sentido de contemplar as necessidades subjetivas e não espontâneas desses idosos, apreendendo os elementos da cultura corporal construídos durante sua vida e direcionar as práticas a promoção da saúde, potencializando esses espaços, ou seja, a ampliação do utilitarismo do binômio exercício físico/ saúde direcionado a aspectos puramente fisiológicos tende a ser ultrapassada.

\section{REFERÊNCIAS}

ANJOS, T. C.; DUARTE, A. C. G. O. A Educação Física e a Estratégia de Saúde da Família: formação e atuação profissional. Physis Revista de Saúde Coletiva, Rio de Janeiro, v. 19, n. 4, p. 1127-1144, out./dez. 2009.

BARDIN, L. Análise de Conteúdo. Lisboa, Portugal: Edições 70, 1977.

BEZERRA, A. F. B.; ESPIRITO SANTO, A. C. G.; BATISTA FILHO, M. Concepções e práticas do agente comunitário na atenção à saúde do idoso. Rev. Saúde Pública. São Paulo, v. 39, n. 5, p. 80915, out. 2005.

BRASIL. Ministério da Saúde. Portaria $\mathbf{n}^{\mathbf{0}}$. 154 de 24 de janeiro de 2008. Cria os Núcleos de Apoio a Saúde da Família. Brasília: Ministério da Saúde; 2008.

CAMPOS, F. E.; BELISÁRIO, S. A. O Programa de Saúde da Família e os desafios para a formação profissional e a educação continuada. Interface - Comunicação, Saúde, Educação, Botucatu, v. 5, n. 9, p. 133-142, ago. 2001.

CZERESNIA, D. The concept of health and the difference between prevention and promotion. Cadernos de Saúde Pública, Rio de Janeiro, v 15, n. 4, p. 701-709, out./dez. 1999.

DIOGO M. J. D'E. Formação de recursos humanos na área da saúde do idoso. Rev Latino-am Enfermagem, v. 12, n. 2, p. 280-282, mar./abr. 2004.

GIL, A. Métodos e técnicas de pesquisa social. São Paulo: Atlas, 1994.

LUZ, M. T. Educação Física e saúde coletiva: papel estratégico da área e possibilidades quanto ao ensino na graduação e integração na rede de serviços públicos de saúde. In: FRAGA, A.B. e WACHS, F. Educação Física e Saúde Coletiva. Políticas de Formação 
e Perspectivas de Intervenção. Porto Alegre: Editora da UFRS, 2007.

MACHADO, M. F. A. S., et. al. Integralidade, formação de saúde, educação em saúde e as propostas do SUS - uma revisão conceitual. Ciência \& Saúde Coletiva, Rio de Janeiro, v. 12, n. 2, p. 335-342, abr. 2007.

MALTA, D. C. et al.A Política Nacional de Promoção da Saúde e a agenda da atividade física no contexto do SUS. Epidemiol. Serv. Saúde, Brasília, v. 18 n. 1, p. 79-86, jan./mar. 2009.

MATTOS, R. A. A integralidade na prática (ou sobre a prática da integralidade). Cadernos de Saúde Pública, Rio de Janeiro, v. 20, n. 5, p. 1411-1416, set./ out. 2004.

MINAYO, M. C. O desafio do conhecimento: pesquisa qualitativa em saúde. Rio de Janeiro: Hucitec; Abrasco, 1993.

MIRANDA, L. M. de; FARIAS, S. F. As contribuições da internet para o idoso: uma revisão de literatura. Interface - Comunicação, Saúde, Educação, Botucatu, v.13, n.29, p.383-94, abr./ jun. 2009.

MINAYO, M.C.S. Pesquisa Social: teoria, método e criatividade. Rio de janeiro: Vozes. p, 09 - 30, 1994.

MOTTA, L. B.; CALDAS, C. P.; ASSIS, M. A formação de profissionais para a atenção integral à saúde do idoso: a experiência interdisciplinar do NAI - UNATI/UERJ. Ciência \& Saúde Coletiva, Rio de Janeiro, v. 13, n. 4, p. 1143-1151, ago. 2008.

NEGREIROS, T. C. G. M. Espiritualidade: desejo de eternidade ou sinal de maturidade? Revista Mal-Estar e
Subjetividade. Fortaleza, v. 3, n. 2, p. 275-291, set. 2003

RAMOS, M. P. Apoio social e saúde entre Idosos. Sociologias, Porto Alegre, v. 7 , n. 4, p. 156-175, jun. 2002.

RODRIGUES, J. C.; ARAUJO, C. L. O. Análise do Conhecimento e prática dos Agentes Comunitários de Saúde na Saúde do Idoso. Estud. interdiscipl. envelhec., Porto Alegre, v. 15, n. 1, p. 117-127, jan./jun.2010.

SANTANA, M. S.; MAIA, E. M. C. Atividade Física e Bem-Estar na Velhice. Rev. salud pública, Bogotá, v. 11, n. 2, p. 225-236, mar./abr. 2009

SILVA, D. J.; ROS, M. A. Inserção de profissionais de fisioterapia na equipe de saúde da família e Sistema Único de Saúde: desafios na formação. Ciência \& Saúde Coletiva, Rio de Janeiro, v. 12, n. 6, p. 1673-1681, nov./dez. 2007.

SILVESTRE, J. A.; COSTA NETO, M. M. Abordagem do idoso em programas de saúde da família. Cadernos de Saúde Pública, Rio de Janeiro, v. 19, n. 3, p. 839-847, jun. 2003. 
ANALYSIS OF SENSE OF OLDER MEMBERS OF PRACTICE GROUP OF BODY IN PRIMARY: Motivational aspects and make multiprofessional

\section{ABSTRACT}

The objective of this study was to analyze the perceptions of the elderly, about the motivational aspects and significance / importance of bodily practices, as well as the work developed by multidisciplinary health team. The study is characterized as descriptive and exploratory qualitative approach, which used the method of content analysis. We interviewed 14 elderly. Therapeutic measures, linked to some positive outcomes and health promotion in general were the main reasons listed. As noted in making multi-interdisciplinary approach is ineffective. Thus, a reinterpretation of these spaces is needed.

Keywords: Exercise; Health Promotion; Health of the Elderly.

Recebido em: março/2012

Aprovado em: julho/2012 